\title{
A Comparison of Fused Segmentation Algorithms for Iris Verification
}

\author{
Yasiel Sanchez-Gonzalez, Yasser Chacon-Cabrera, and Eduardo Garea-Llano \\ Advanced Technologies Application Center, \\ 7 ma A $\sharp 21406$, Playa, Havana 12200, Cuba \\ \{ysanchez, ychacon, egarea\}@cenatav.co.cu
}

\begin{abstract}
Recent studies show fusion at level of segmentation to be useful for more robust iris recognition rates compared with simple segmentation. In this paper we perform Sum-Rule Interpolation at level of the result of the normalized segmented iris images using the well-known Daugman's algorithm, since the process of normalization is essentially composed by two parts: Iris segmentation, in which the pupillary and limbic polar curves are detected and Iris normalization: a normalized representation of the iris texture is created using angular and pupil-tolimbic radial coordinates. For evaluation we propose an experimental fusion scheme using three automatic segmentation algorithms which have reported good results and are not computationally expensive. The experiments were performed on the CASIA V3-Interval, CASIA.V4-Thousand and UBIRIS V1 datasets showing increased recognition accuracy for representative feature extraction algorithms.
\end{abstract}

Keywords: segmentation fusion, iris verification.

\section{Introduction}

The biometric iris recognition has had in recent years a significant development. The iris is considered one of the best biometric features from the high randomness of the texture pattern that composes it. Recognition or verification of a person by iris constitutes one of the main applications of the biometrics at present time. The first step in the recognition process is the iris localization or segmentation. The iris is characterized by a quasi-circular form limited by two borders (iris inner border and outer). The iris inner border coincides with the contour of the pupil. Many algorithms have been proposed for automatic iris contour segmentation to obtain, the iris inner and outer border parameters in order to obtain the iris texture information 1 .

Clearly, the demand of large-scale applications requires a high precision as possible to avoid potential misclassification. It is widely known that the performance of cooperative biometric systems based on the standardization and control of the conditions under which the images were taken are different from applications implemented in less controlled settings. This fact has led to intensive research activity in the last 10 years which has resulted in the proposals for

E. Bayro-Corrochano and E. Hancock (Eds.): CIARP 2014, LNCS 8827, pp. 112-119 2014.

(C) Springer International Publishing Switzerland 2014 
improvement of preprocessing and segmentation algorithms. These techniques have a fundamental role in the recognition process because of its dependence on the quality of the images.

The combination of biometric information can increase the accuracy at the cost of additional resources and it is traditionally used in the classification or in the decision level. However, these strategies of fusion have been presented limitations, since a number of algorithms can be dedicated to perform similar processing which leads to an increased computational cost. The existence of conflicting information from various processing algorithms can reduce system performance.

In 2 was introduced the concept of multi-segmentation fusion to combine separate iris segmentation results. The authors experimentally demonstrated that the recognition accuracy for representative algorithms increases efficacy in the CASIA.V4-Interval database, when the results of two manual segmentations are combined, but not results of the combination of more than two segmentation or results from the combination of automatic segmentation were explored, so it is not really clear which is the final effect on the recognition accuracy produced by the nature of the algorithms used in the segmentation.

The remainder of this paper is organized as follows. Section 2 presents the proposed segmentation model: Section 3 presents a short description of the experimented segmentation algorithms. Section 4 introduces experimental setup and fusion scheme. Section 5 presents the results and discussion of the experimental evaluation. Finally, Section 6 concludes this work.

\section{Fusion Segmentation Model}

In 2 the fusion of multiple segmentation results $S 1, S 2, \ldots, S k$ of the same input iris image using multiple segmentation algorithms was proposed as step into iris processing between image capture and normalization. Taking into account that not all iris feature extraction techniques require the same preprocessing tasks, the authors proposed the use of Daugman's normalization method [3], which is the basis for most commercial applications.

They explored the idea of minimizing the effect of mapping deformations due to inaccurately localized boundaries in the rubbersheet transform by a better pupillary $(\mathrm{P})$ and limbic $(\mathrm{L})$ representation. For the fusion task they introduced two techniques [2]:

Sum-Rule Interpolation: The rule combines the obtained boundaries $B_{1}, B_{2}, B_{k}$ into a single boundary $B$ by the arithmetic mean of sampled boundaries. The interpolation is performed for $B=P$ and $B=L$ separately.The method can be applied to interpolate between approximations of the upper and lower eye lids to derive a common noise mask.

Augmented-Model Interpolation: Authors proposed a re-parameterization of boundary curves as an alternative approach to their fusion. They proposed fitting a model to the union of sampled edge points, to avoid the problem of not 
equally spaced sampling interval of the curves $[0,2 \pi]$ in the case when limits are quite different.

The authors assess its performance on manually segmented iris images, to avoid any dependencies between segmentation algorithms enabling a fair test of the fusion rule, they were based in the idea that, if fusion improves the manual segmentation it is also positive the improvement of results of automatic segmentation techniques, but they do not explore the effect which is produced by the fusion when combining the results of automated segmentation algorithms.

Taken into account that there are not great differences between the Sum Rule Interpolation and the Augmented Model Interpolation [2], we perform our experiment using the first one. We used normalized segmented iris images Eq.1 by the Daugman's normalization algorithm 3 .

$$
\text { SumRule }: T(S):=\frac{1}{k} \sum_{i=1}^{k} T_{i}\left(S_{i}\right),
$$

where $T_{1}\left(S_{1}\right), T_{2}\left(S_{2}\right), \ldots, T_{K}\left(S_{k}\right)$ are the sampled normalized textures obtained by different segmentation methods and $T(S)$ is the arithmetic mean of sampled textures.

On this basis, in this paper we propose an experimental fusion scheme using three automatic segmentation algorithms that have reported good results and are not computationally expensive.

\section{Experimented Iris Segmentation Algorithms}

The iris segmentation methods are oriented toward isolating the iris of an image. This is usually performed in two steps: first the inner and outer boundaries of the iris region are estimated, and then the occlusions and reflections are detected and removed. Both, the inner boundary and the outer boundary of a typical iris can approximately be taken as circles.

In the literature there are a lot of iris segmentation algorithms, but for this work we have chosen three of the most used in experimental software free to use.

\subsection{Hough Transform Based Segmentation Algorithms}

Hough transform is a standard image analysis tool for finding curves that can be defined in a parametrical form such as lines, polynomials and circles. The recognition of a global pattern is achieved using the local patterns. For instance, recognition of a circle can be achieved by considering the strong edges in an image as the local patterns and searching for the maximum value of a circular Hough transform.

Many authors use the Hough transform such as [4, [5] and [6]. For example in [6], an edge map of the image is obtained using the magnitude of the image intensity gradient. The edge map is then used in a voting process to maximize the defined Hough transform for the desired contour. The limbus and pupil are modeled as circles. 


\subsection{Contrast-Adjusted Hough Transform (S1)}

Contrast-adjusted Hough Transform (CHT), is the iris segmentation algorithm implemented in the USIT toolbox 1 . It is based on a Masek 7] implementation of a Hough Transform approach using (database-specific) contrast adjustment to enhance pupillary and limbic boundaries, Canny edge detection to detect boundary curves, and enhancement techniques to remove unlikely edges.

\subsection{Weighted Adaptive Hough and Ellipsopolar Transform (S2)}

Weighted Adaptive Hough and Ellipsopolar Transforms (WHT) [8], the iris segmentation algorithm implemented in the USIT toolbox, based on a weighted adaptive Hough transform, iteratively refining a ROI to find an initial center point, which is used to polar transform the image and extract polar and limbic boundary curves one after another from an ellipso-polar representation.

\subsection{Viterbi-Based Segmentation Algorithm (S3)}

The open source reference system OSIRIS, version v4.12, in the segmentation part uses a Viterbi algorithm based iris segmentation algorithm 9 to find the iris and pupil boundaries.

The first step of the segmentation approach consists in a rough localization of the pupil area. First, filling the white holes removes specular reflections due to illuminators. Then, a morphological opening removes dark areas smaller than the disk-shaped structuring element. Then, the pupil area is almost the biggest dark area, and is surrounded by the iris, which is darker than the sclera and the skin.

Consequently the sum of intensity values in large windows in the image is computed, and the minimum corresponds to the pupil area. The pupil being roughly located, a morphological reconstruction allows estimating a first center, which is required for exploiting the Viterbi algorithm. The second step consists in accurately extracting the pupil contour and a well estimated pupil circle for normalization. Relying on the pupil center, the Viterbi algorithm is used to extract the accurate pupil contour. This accurate contour will be used to build the iris mask for recognition purposes.

\section{Experimental Design}

The basic idea of the proposed experimental scheme is shown in Fig 1, The principal segmentation scheme consists in the combination of two and three segmentation algorithms described above. The objective is to compare the performance of verification task when the results of segmentation algorithms are fused in four different combinations $(S 1-S 2),(S 1-S 3),(S 2-S 3)$ and $(S 1-S 2-S 3)$ that are all possible combination between them.

\footnotetext{
${ }^{1}$ USIT - University of Salzburg Iris Toolkit v1.0 http://www. wavelab.at/sources/

${ }^{2}$ OSIRIS, version v4.1, http://svnext.it-sudparis.eu/svnview2-eph/ref_syst/Iris_Osiris/
} 


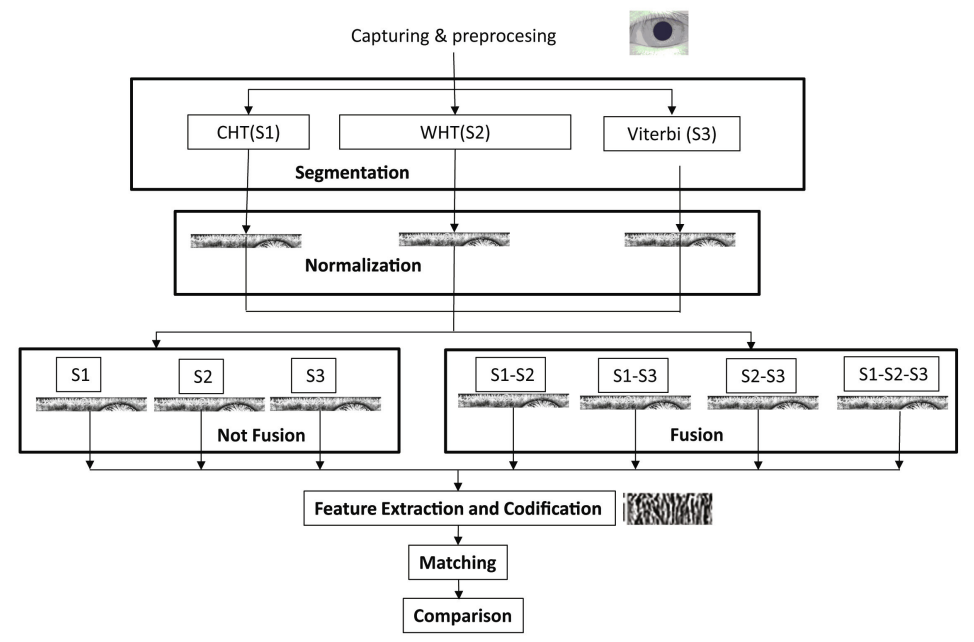

Fig. 1. Experimental scheme for comparison of iris segmentation methods for fusion at segmentation stage

For experiments we employ the iris databases CASIA-V3-Interval, CASIAV4-Thousand, and UBIRIS V1, see Fig,2,

The CASIA-V3-Interva 3 dataset is composed of high quality NIR illuminated indoor images with $320 \times 280$ pixel resolution (2639 images, 395 classes). For the experiments we used the whole dataset.

CASIA.v4-Thousand ${ }^{3}$ contains 20,000 iris images from 1,000 subjects. The main sources of intra-class variations in CASIA-Iris-Thousand are eyeglasses and specular reflections. For the experiments we used a subset composed by 3104 images from the all subjects.

UBIRIS.v1[10] dataset is comprised of 1877 images collected from 241 persons in two distinct sessions. This database incorporates images with several noise factors, simulating less constrained image acquisition environments. For the experiments we used a subset composed by 771 images from all the subjects.

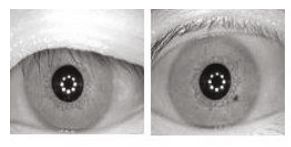

a)

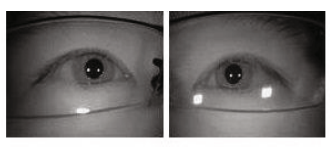

b)

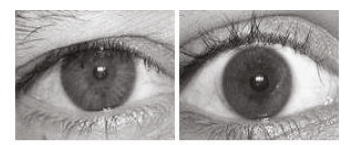

c)

Fig. 2. Examples of the experimented datasets a) CASIA-V3-Interval, b)CASIA- V4Thousand c) UBIRIS V1

3 CASIA-V3-Interval, CASIA V4- Thousands. The Center of Biometrics and Security Research, CASIA Iris Image Database, http://biometrics.idealtest.org/ 
For feature extraction we used four well known methods. Daugman [3, is the classic feature extraction method which uses a 2D version of Gabor filters in order to encode iris pattern. It demodulated the output of the Gabor filters by quantizing the phase information into four levels. These four levels were represented by two bits of data, so that each pixel corresponds to two bits of data in the iris template.

Masek [7], is a feature extraction method which convolved the normalized iris pattern with 1D Log-Gabor wavelets.

$\mathrm{Ma}$ [1], extracts sharp variations of intensity signals. The normalized iris texture is divided into stripes to obtain 10 one dimensional signals, each one averaged from the pixels of 5 adjacent rows. Subsequently, a 1-D wavelet transform is applied to each of the ten 1-D intensity signals. Detected minima and maxima from two specific subbands serve as features where sequences of 1 s and 0 s are assigned to the iris-code until new maxima or minima are found.

Monro 12, started from a general paradigm where feature vectors will be derived from the zero crossings of the differences between 1D DCT coefficients calculated in rectangular image patches.

\section{$5 \quad$ Experimental Results}

The evaluation of segmentation performance and accuracy was assessed by the degree of influence on verification recognition accuracy. It was estimated by ROC curves; False Acceptance Rate $(F A R)$ versus Genuine Acceptance Rate $(G A R)$. Tables 1, 2 and 3 report the results of the GAR and Equal Error Rate $(E R R)$ for each of automatic segmentation and fused results.

Table 1. Recognition accuracy in $G A R$ and $E E R$ at $\leq 0.01 \%$ FAR (CASIA V3-Int.)

\begin{tabular}{|c|c|c|c|c|c|c|c|c|}
\hline$\overline{\text { Algortihm }}$ & $\begin{array}{l}\text { Daugm } \\
\text { GAR(\%) }\end{array}$ & $\begin{array}{l}\text { an } \\
\operatorname{EER}(\%)\end{array}$ & $\begin{array}{l}\text { Masek } \\
\text { GAR(\%) }\end{array}$ & $\operatorname{EER}(\%)$ & $\begin{array}{l}\text { Ma } \\
\text { GAR(\%) }\end{array}$ & $\operatorname{EER}(\%)$ & $\begin{array}{l}\text { Monro } \\
\text { GAR(\%) }\end{array}$ & $\operatorname{EER}(\%)$ \\
\hline S1 & 98.5 & 1.27 & 92.5 & 5.90 & 96.6 & 2.62 & 98.4 & 1.44 \\
\hline$\overline{\mathrm{S} 2}$ & 97.8 & 1.95 & 92.5 & 6.32 & 95.6 & 2.85 & 96.9 & 2.55 \\
\hline$\overline{\mathrm{S} 3}$ & 99.2 & 0.76 & 93.2 & 5.90 & 98.4 & 1.27 & 98.7 & 1.37 \\
\hline$\overline{\text { S1-S2 }}$ & 99.3 & 0.86 & 93.9 & 4.80 & 98.2 & 1.76 & 98.6 & 1.44 \\
\hline S1-S3 & 98.9 & 1.11 & 93.2 & 5.29 & 97.7 & 2.02 & 98.6 & 1.34 \\
\hline S2-S3 & 98.0 & 1.85 & 92.8 & 5.32 & 97.4 & 2.27 & 97.4 & 2.27 \\
\hline$\overline{\mathrm{S} 1-S 2-}$ S3 & 99.8 & 0.66 & 94.0 & 4.87 & 98.7 & 1.14 & 98.9 & 1.34 \\
\hline
\end{tabular}

Under conditions of CASIA-V3-Interval and CASIA-V4-Thousands datasets (Table 1 and 2), segmentation 3 (Viterbi) obtained the best results in all cases with $G A R=92,5-99,2 \%$ for Daugman, $=90,2-93,2 \%$, for Masek, $=87,1-$ $98,4 \%$ for $\mathrm{Ma}$ and $=75,1-98,7 \%$ for Monro at $F A R \leq 0,01 \%$. These results suggest that segmentation 3 is more accurate for CASIA datasets conditions.

For UBIRIS V1 dataset (Table 3), segmentation 1 (CHT) obtained the best results in all cases with $G A R=92,2 \%$ for Daugman, $=92,5 \%$ for Masek, $=91,8 \%$ 
Table 2. Recognition accuracy in $G A R$ and $E E R$ at $\leq 0.01 \%$ FAR (CASIA V4-Th.)

\begin{tabular}{|c|c|c|c|c|c|c|c|c|}
\hline Algortihm & $\begin{array}{l}\text { Daugm } \\
\operatorname{GAR}(\%)\end{array}$ & an $\operatorname{EER}(\%)$ & $\begin{array}{l}\text { Masek } \\
\text { GAR }(\%)\end{array}$ & $\operatorname{EER}(\%)$ & $\begin{array}{l}\text { Ma } \\
\text { GAR }(\%)\end{array}$ & $\operatorname{EER}(\%)$ & $\begin{array}{l}\text { Monro } \\
\text { GAR(\%) }\end{array}$ & $\operatorname{EER}(\%)$ \\
\hline$\overline{\mathrm{S} 1}$ & 86.4 & 99.68 & 86.2 & 10.9 & 79.1 & 15.10 & 67.1 & 18.45 \\
\hline$\overline{\mathrm{S} 2}$ & 88.6 & 7.86 & 87.2 & 7.86 & 82.8 & 11.6 & 72.5 & 15.02 \\
\hline$\overline{\text { S3 }}$ & 92.5 & 5.03 & 90.2 & 5.91 & 87.1 & 7.85 & 75.1 & 14.42 \\
\hline S1-S2 & 96.8 & 2.59 & 94.0 & 4.05 & 89.7 & 7.79 & 75.2 & 9.42 \\
\hline$\overline{\text { S1-S3 }}$ & 94.9 & 3.53 & 93.5 & 4.23 & 87.8 & 8.68 & 76.1 & 15.09 \\
\hline$\overline{\mathrm{S} 2-\mathrm{S} 3}$ & 93.5 & 3.39 & 93.2 & 4.30 & 88.7 & 7.96 & 80.5 & 10.90 \\
\hline$\overline{\mathrm{S} 1-\mathrm{S} 2-\mathrm{S} 3}$ & 96.7 & 2.73 & 94.1 & 3.68 & 90.6 & 7.64 & 89.0 & 6.35 \\
\hline
\end{tabular}

Table 3. Recognition accuracy in $G A R$ and $E E R$ at $\leq 0.01 \%$ FAR (UBIRIS V1.)

\begin{tabular}{|c|c|c|c|c|c|c|c|c|}
\hline Algortihm & $\begin{array}{l}\text { Daugm } \\
\operatorname{GAR}(\%)\end{array}$ & an $\operatorname{EER}(\%)$ & $\begin{array}{l}\text { Masek } \\
\operatorname{GAR}(\%)\end{array}$ & $\operatorname{EER}(\%)$ & $\begin{array}{l}\text { Ma } \\
\operatorname{GAR}(\%)\end{array}$ & $\operatorname{EER}(\%)$ & $\begin{array}{l}\text { Monro } \\
\text { GAR(\%) }\end{array}$ & $\operatorname{EER}(\%)$ \\
\hline$\overline{\mathrm{S} 1}$ & 92.2 & 6.04 & 92.5 & 5.70 & 91.8 & 6.88 & 91.4 & 5.36 \\
\hline S2 & 89.0 & 9.80 & 83.5 & 4.27 & 89.7 & 8.28 & 88.4 & 9.80 \\
\hline$\overline{\text { S3 }}$ & 89.5 & 8.26 & 88.9 & 9.26 & 87.6 & 10.12 & 85.5 & 9.55 \\
\hline S1-S2 & 97.2 & 2.80 & 97.2 & 1.20 & $\begin{array}{l}99.1 \\
\end{array}$ & 1.20 & 97.2 & 2.04 \\
\hline$\overline{\text { S1-S3 }}$ & 95.1 & 4.12 & 95.1 & 3.20 & 94.6 & 4.51 & 93.4 & 4.52 \\
\hline S2-S3 & 90.9 & 6.68 & 91.6 & 6.60 & 90.1 & 6.68 & 90.1 & 8.52 \\
\hline$\overline{\mathrm{S} 1-\mathrm{S} 2-\mathrm{S} 3}$ & 98.0 & 1.33 & 98.9 & 1.33 & 99.0 & 1.33 & 96.9 & 2.26 \\
\hline
\end{tabular}

for $\mathrm{Ma}$ and $=91,4 \%$ for Monro at $F A R \leq 0,01 \%$. These results suggest that segmentation 1 is more accurate for UBIRIS V1 dataset conditions.

Results in the three tables shows the positive impact of fused segmentation results on the accuracy in the recognition. It is observed that in all cases the GAR increased and EER decreased compared with the results of the verification when a single segmentation algorithm is used. In this case the combination of the three evaluated algorithms produce an increase in GAR with the most significant increase in $96,7-99,8 \%$ (CASIA) for Daugman and an EER of $0,66-2,73 \%$ and $G A R=99 \%$ (UBIRIS) with an $E E R=1,33 \%$ for Ma.

\section{Conclusions}

From the proven idea that fusion at segmentation level improves iris recognition rates [2, in this paper we perform a comparative study at level of the result of the normalized segmented iris images. We experimented the fusion of three automatic segmentation algorithms comparing the performance of verification task when in segmentation stage is used each segmentation algorithm separately and when their results are fused in four different combinations using the SumRule Interpolation. Evaluations using automatic segmentations on three datasets showed improvement by segmentation fusion for each of the employed feature extraction algorithms. 
Future works will focus on the fusion of other segmentation algorithms combining circular and non-circular boundaries and on new fusion strategies using ensemble clustering methods.

\section{References}

1. Connaughton, R., Bowyer, K.W., Flynn, P.J.: Fusion of face and iris biometrics. In: Handbook of Iris Recognition, pp. 219-237. Springer (2013)

2. Uhl, A., Wild, P.: Fusion of iris segmentation results. In: Ruiz-Shulcloper, J., Sanniti di Baja, G. (eds.) CIARP 2013, Part II. LNCS, vol. 8259, pp. 310-317. Springer, Heidelberg (2013)

3. Daugman, J.G.: High confidence visual recognition of persons by a test of statistical independence. IEEE Transactions on Pattern Analysis and Machine Intelligence 15(11), 1148-1161 (1993)

4. Kashima, H., Hongo, H., Kato, K., Yamamoto, K.: An iris detection method using the hough transform and its evaluation for facial and eye movement. In: The 5th Asian Conference on Computer Vision, pp. 23-25 (2002)

5. Cherabit, N., Chelali, F.Z., Djeradi, A.: Circular hough transform for iris localization. Science and Technology 2(5), 114-121 (2012)

6. Wildes, R.P.: Iris recognition: an emerging biometric technology. Proceedings of the IEEE 85(9), 1348-1363 (1997)

7. Masek, L.: Recognition of human iris patterns for biometric identification. Technical report (2003)

8. Uhl, A., Wild, P.: Weighted adaptive hough and ellipsopolar transforms for realtime iris segmentation. In: 2012 5th IAPR International Conference on Biometrics (ICB), pp. 283-290. IEEE (2012)

9. Sutra, G., Garcia-Salicetti, S., Dorizzi, B.: The viterbi algorithm at different resolutions for enhanced iris segmentation. In: 2012 5th IAPR International Conference on Biometrics (ICB), pp. 310-316. IEEE (2012)

10. Proença, H., Alexandre, L.A.: UBIRIS: A noisy iris image database. In: Roli, F., Vitulano, S. (eds.) ICIAP 2005. LNCS, vol. 3617, pp. 970-977. Springer, Heidelberg (2005)

11. Ma, L., Tan, T., Wang, Y., Zhang, D.: Personal identification based on iris texture analysis. IEEE Transactions on Pattern Analysis and Machine Intelligence 25(12), 1519-1533 (2003)

12. Monro, D.M., Rakshit, S., Dexin, Z.: Dct-based iris recognition. IEEE Transactions on Pattern Analysis and Machine Intelligence 29(4), 586-595 (2007) 